\title{
On-line Monitoring of Transformer Oil Degradation Based on Fiber Optic Sensors
}

\author{
Tae-Young Kim*, Jong-Eun Kim and Kwang S. Suh \\ Department of Materials Science and Engineering, Korea University \\ 5-1 Anam-dong, Seongbuk-gu, Seoul 137-713, Korea \\ (received July 25, 2007; accepted July 16, 2008)
}

Key words: fiber optic sensors, acoustic monitoring, insulating oil, degradation

In this paper, we present the preliminary results of our study on the use of a fiber optic sensor as a diagnostic and monitoring tool for evaluating the condition of transformer oil. An all-optical-fiber sensing system using a Mach-Zehnder interferometry technique was constructed to monitor acoustic wave propagation properties in differently aged transformer oil. It was found that the measured sound attenuation in the oil varies as it degrades, suggesting that the observation of sound attenuation with the fiber optic sensor can indicate the condition of service-aged transformer oil.

\section{Introduction}

The in-service failure of transformers used in electric power distribution has been reported over several decades to be potentially dangerous to utility personnel through explosions and fire, potentially damaging to the environment through oil leakage and resulting in a significant increase in operating costs. ${ }^{(1-3)}$ As transformers age, their insulation strength often degrades to the point that they cannot withstand an electrical breakdown or other incidents, resulting in a higher probability of catastrophic failures. ${ }^{(4-6)}$ The quality of oil in a transformer plays an important role in its performance and service life since the degradation of the oil increases the risk of earlier failures. ${ }^{(5,7)}$ Hence, there is an increasing need for diagnostic and monitoring tools that assess the condition of the transformer oil and estimate its ultimate service life. ${ }^{(8-10)}$

Conventionally, the evaluation of the condition of transformer oil is carried out by a method of analyzing the acidity, applying a breakdown voltage, and analyzing the resulting gas at the laboratory. However, these tests are only performed for sample materials without testing them immediately on the spot, and therefore appropriate action cannot be taken before failures occur through a faster-than-normal aging process.

Effective diagnostics are those that can be carried out without interrupting the operation of the equipment so that if there is a problem, the transformer oil can be replaced before it fails. In light of continuous on-line monitoring techniques, the use

*Corresponding author: e-mail: thomas75@korea.ac.kr 
of acoustic sensors such as piezoelectric and fiber optic sensors is expected to increase for the evaluation of the condition of transformers. In practice, these acoustic sensors are used to detect the acoustic signal from a partial discharge in a transformer while they are mounted either on the transformer tank wall or in the oil inside the tank. ${ }^{(11-13)}$ A fiber optic sensor has advantages over a piezoelectric sensor in that the latter is typically placed on the outside of the tank wall and its external location limits sensitivity because of the attenuation of the signal at the oil-to-tank and tank-to-sensor interfaces. On the other hand, fiber optic sensors can be installed in the transformer oil, thus improving sensitivity and eliminating the signal interference problem. In addition to the use of fiber optic sensors for the detection of a partial discharge, these sensors can also be used to assess the condition of transformer oil in the same way that they detect the variation of acoustic wave attenuation in the aged oil.

This work is a preliminary study on the application of fiber optic sensors for monitoring and evaluating the condition of transformer oil. Acoustic attenuation measured using fiber optic sensors will be discussed in terms of the condition of aging transformer oil.

\section{Materials and Method}

Figure 1(a) illustrates the fiber optic sensor system used in this study. An all-opticalfiber-sensing system was constructed by employing Mach-Zehnder interferometry. This system involves a sensing mechanism for altering such properties as refractive index and path length when its sensing arm is within the acoustic region. Consequently, the relative phase-change difference produced by two arms (one a reference arm and the other in the acoustic region) is converted into an intensity signal when the lights in the two arms are combined. The system is composed of a $632.8 \mathrm{~nm}$ He-Ne laser, a single-mode fiber, a photodetector and a digitizing oscilloscope. The He-Ne laser was used as a light source and a single-mode fiber, which has been shown to be more sensitive than a multimode fiber, was used to transmit the light. A polarization controller was used to prevent the signal from fading due to attenuation, which is mainly caused by the difference in the polarization state of the light in the two arms. The sensing part was coiled 40 times so that an acoustic signal incident on the sensing coil will affect every turn of the coil as the acoustic wave passes the coil. Even though the phase shift per turn is small, the total change induced by a number of turns in the coil is sufficient to introduce a measurable optical phase shift caused by the travelling acoustic wave in the fluid medium. As is shown in Fig. 1(a), the sensing coil was made in the form of a spiral with inner and outer diameters of 0.5 and $4 \mathrm{~cm}$, respectively. Since the upper limit for the coil thickness is equal to one-half the wavelength of the acoustic waves to be detected, the sensing coil was suitable for sensing acoustic waves with frequencies in the approximate range of $1-$ $150 \mathrm{kHz}$. The measured signal is converted into an electrical signal by the photodetector and transmitted to a high-pass filter whose cutoff frequency is $1 \mathrm{kHz}$ to prevent signal drift. After amplification using a wide-band preamplifier, the detected signal was obtained using the digitizing oscilloscope.

A test cell was made of a transparent polyacrylate tank $\left(600 \times 150 \times 300 \mathrm{~mm}^{3}\right)$. The test cell contains mineral transformer oil in which the acoustic wave emitter and the 


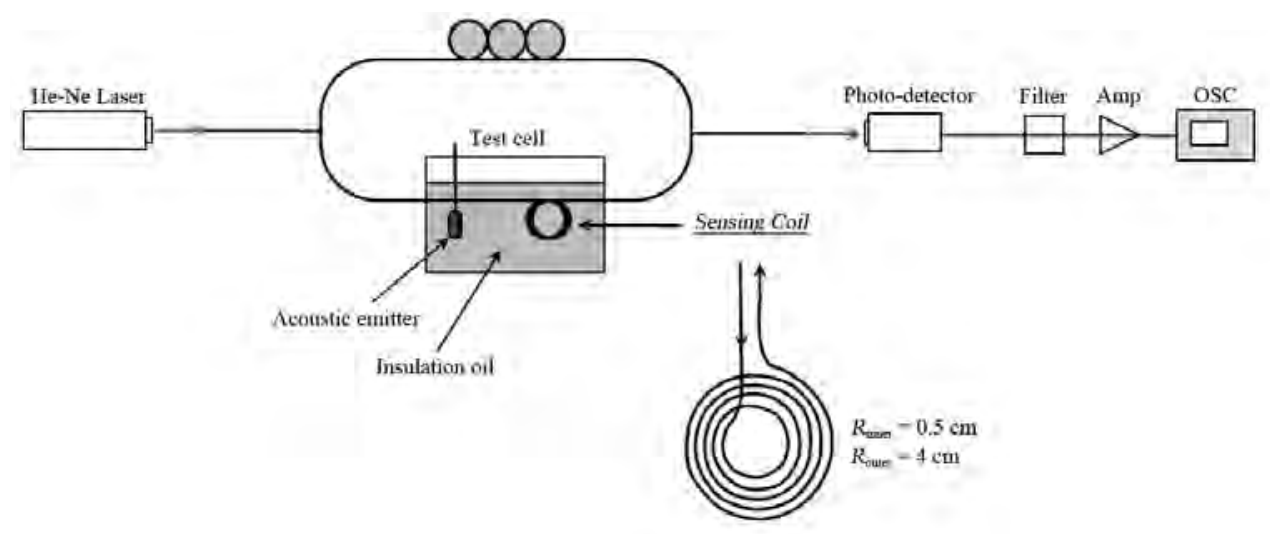

(a)

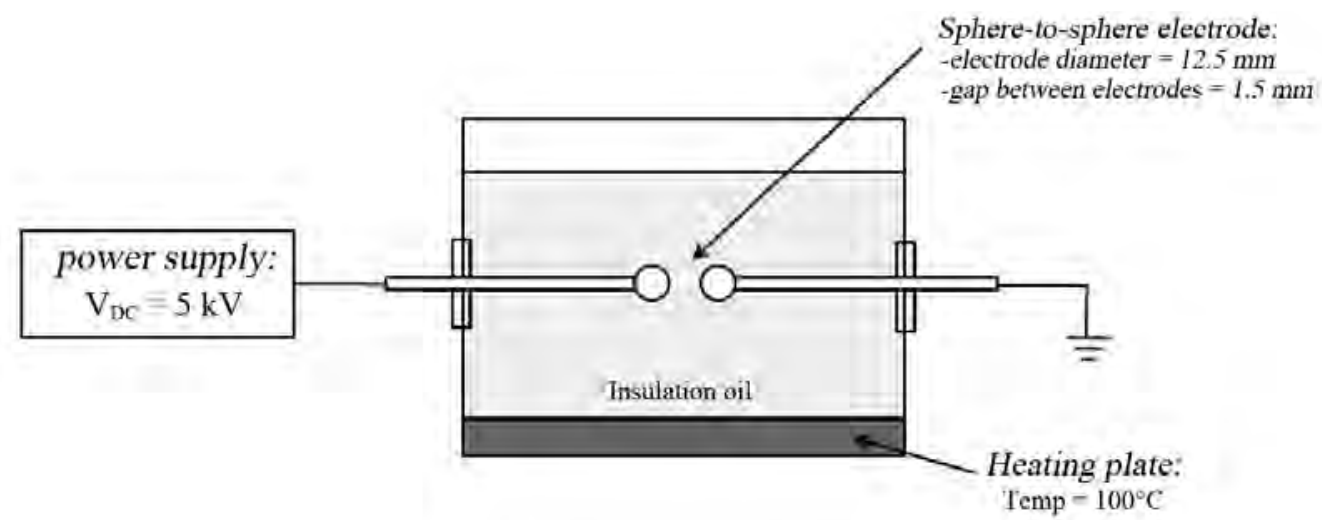

(b)

Fig. 1. (a) Schematic configuration of fiber optic sensor system and (b) apparatus for accelerated oil degradation.

sensing coils of the fiber optic sensor system are placed. The acoustic emitter, made of a piezoelectric ceramic disk with a diameter of $1.5 \mathrm{~cm}$, was connected to the function generator and set to be movable so that the distance between the acoustic source and the sensor could be adjusted. An acoustic absorber was attached to the inside wall of the test cell to prevent the internal reflection of acoustic waves. A piezoelectric sensor was also mounted near the fiber optic sensor coil to compare the responses of sensors to the acoustic wave propagating from the acoustic emitter. To establish the relationship between the condition of the transformer oil and the response of the fiber optic sensor, it was necessary to perform the tests in differently aged oil samples. For this purpose, an accelerated oil aging experiment was carried out by installing a sphere-sphere electrode system in an oil-filled tank that was then activated by a DC voltage of $5 \mathrm{kV}$ to produce repated arcing events in the oil. With the increasing number of discharging events, the transformer oil degraded and some of the aged oils were taken as samples and subjected to further tests. 


\section{Results and Discussion}

To confirm that the fiber optic sensor system in the present study was functioning properly, a comparison of the sensor system with a piezoelectric sensor was performed. An ultrasound source was placed in the test cell filled with transformer oil, and a piezoelectric sensor and a fiber optic sensor coil were positioned at a distance of 0.1 $\mathrm{m}$ from the acoustic emitter. Different types of acoustic waves were generated at the piezoelectric acoustic emitter driven by square and sinusoidal signals from a function generator.

Figure 2(a) shows the square input signal with a pulse width of $300 \mu$ s applied to the acoustic emitter, while Figs. 2(b) and 2(c) show the outputs from the piezoelectric and fiber optic sensors, respectively. Despite slightly different transient and relaxation responses, the two output waveforms are very similar, showing a response at the rising and falling edges of the input signal. Compared with the input signal, both output signals showed a time delay of $60 \mu \mathrm{s}$; this is a distinctive feature of acoustic sensors. Assuming the velocity of acoustic waves in transformer oil to be $1415 \mathrm{~m} / \mathrm{s}$, this value is in good agreement with the location of the sensors.

Figure 3 shows a comparison between the sinusoidal input signal whose frequency is $122 \mathrm{kHz}$ and the corresponding output of the fiber optic sensor. The distance between the acoustic emitter and the fiber sensing coil was $0.3 \mathrm{~m}$ and the input voltage was set to $30 \mathrm{~V}$. The output signal detected by the fiber optic sensor was averaged to remove environmental noise and was found to be fully consistent with the input signal in shape and frequency.

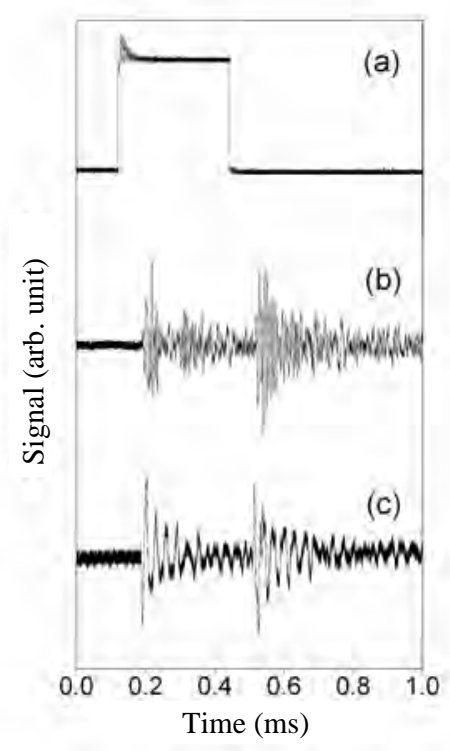

Fig. 2. Response of the sensors to the acoustic signal: (a) input pulse applied to acoustic emitter, (b) output signal from piezoelectric sensor and (c) output signal from fiber optic sensor. 
(a)

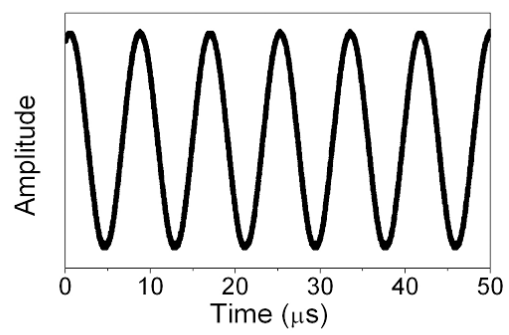

(b)

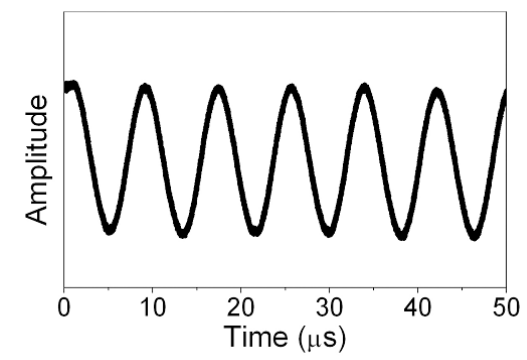

(c)

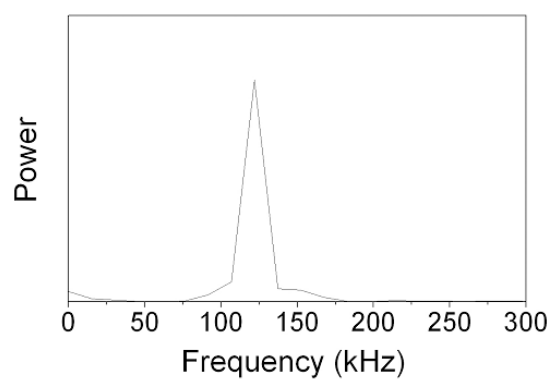

Fig. 3. (a) Sinusoidal signal from function generator, (b) response of fiber optic sensor to the input signal and (c) Fast Fourier transformation (FFT) of the output signal.

The peak-to-peak value of the detected signal in Fig. 3 remained unchanged unless the voltage of the input signal was changed. With the increase in the voltage of the input signal, the signal detected by the fiber optic sensor also increases. Figure 4 shows a good linearity between the peak-to-peak values of the input and output signals.

In Fig. 5, it can be seen that the intensity of the acoustic wave decreases inversely with the distance from the source when a wave propagates through the transformer oil. This is caused by several mechanisms including the acoustic absorption and scattering of the wave in the transformer oil. If any change occurs in the condition of the transformer oil, it is expected to affect the propagation of the acoustic wave, resulting in a change in attenuation phenomena. 


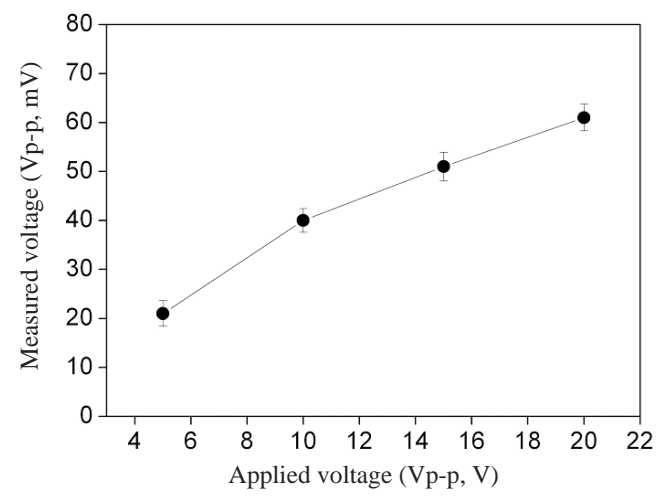

Fig. 4. Plots of output signal versus input signal.

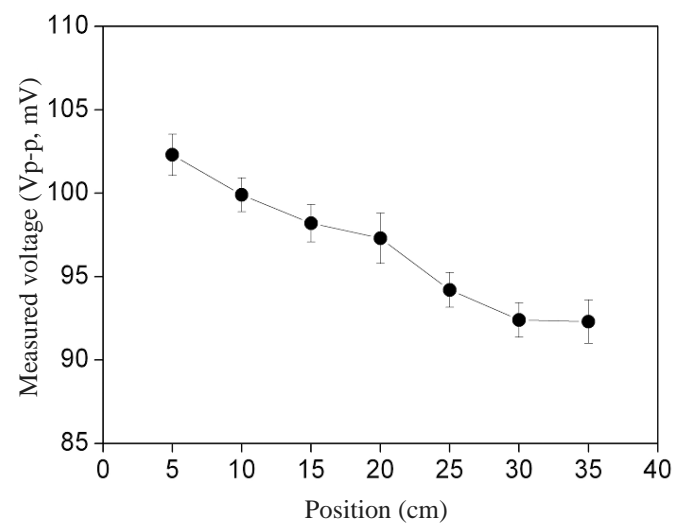

Fig. 5. Sound attenuation characteristic in pure transformer oil.

In most cases, transformer oils are subject to aging under service conditions. Since mineral transformer oils are mixtures of many different hydrocarbon molecules, the aging of transformer oil is associated with a chemical process involving the disruption of the $\mathrm{C}-\mathrm{H}$ and $\mathrm{C}-\mathrm{C}$ bonds of the oil with the formation of new chemical compounds. These by-products range from light gases such as hydrogen and carbon oxides to more complex oxygen-containing and higher-molecular-weight compounds such as alcohols, organic acids and salts of organic acids. Once these defects are produced in oil by the degradation process, the physical parameters of the transformer oil such as viscosity and elasticity are changed. Hence, depending on the condition of the aged transformer oil, the acoustic propagation behavior in the oil is expected to be varied. In view of acoustic wave propagation, when the transformer oil contains gases and particles, the acoustic wave propagates from one medium to another with different values of density and elasticity. Because of the different acoustic impedances of the oil and defects, reflection and refraction at the interface of the two media will take place, resulting in a reduction 
of the energy in the transmitted wave. As the number of these defects increases, the attenuation of the acoustic wave becomes greater. The acoustic diagnostics in the present study with a fiber optic sensor are based on measuring the change in the acoustic wave attenuation, which tracks the degradation process of transformer oil. The amount of the components formed in the oil and the corresponding changes in the acoustic attenuation are the basis for the determination of the oil condition and the extent to which it is affected by the service conditions.

To verify the effect of oil aging on the attenuation of acoustic waves, accelerated oil aging experiments were performed and the aged oil samples were taken according to the number of discharging events, as is shown in Table 1. Then, a sinusoidal acoustic wave of $122 \mathrm{kHz}$ frequency was applied to the acoustic emitter, and the fiber optic sensor was placed in the oil to measure the attenuation of the acoustic wave in differently aged oil samples.

Figure 6 shows a comparison between the acoustic wave attenuation measured using the fiber optic sensor in the differently aged transformer oils. The measured signal was averaged to remove the environmental noise, and the peak-to-peak value was obtained to determine the signal intensity, which is expressed in decibels (dB). In Fig. 6, the signal intensity decreases as a function of distance along the acoustic wave propagation path because of the acoustic energy loss from the acoustic absorption and scattering, which is consistent with the data in Fig. 5. In addition, it was observed that the amount of acoustic attenuation increases as the transformer oil aged. This implies that the repeated arcing events in the oil alter the condition of the transformer oil, which in turn gives rise to a change in its acoustic attenuation properties. From Table 1, it is clear that the content of sludge in the oil increases with aging, which indicates that particles of impurities are formed by the degradation process of oil. These impurities in the oil can act as an obstacle to the acoustic wave propagation, so that the attenuation via acoustic energy loss brought about by scattering becomes greater.

Once these impurities are formed in the oil, they increase its viscosity as is also shown in Table 1, while high-molecular-weight compounds produced by the chemical reactions involved in oil aging also contribute to the increase in viscosity. It has been reported that the acoustic energy loss via acoustic absorption is proportional to and

Table 1

Specifications of aged transformer oils.

\begin{tabular}{ccccc}
\hline $\begin{array}{c}\text { Oil } \\
\text { Sample }\end{array}$ & $\begin{array}{c}\text { Discharging } \\
\text { count }\end{array}$ & $\begin{array}{c}\text { Viscosity (cP) } \\
\text { at } 25^{\circ} \mathrm{C}\end{array}$ & $\begin{array}{c}\text { Moisture } \\
\text { content } \\
(\mathrm{ppm})\end{array}$ & $\begin{array}{c}\text { Sludge } \\
\text { content } \\
(\mathrm{mg} / 100 \mathrm{l})\end{array}$ \\
\hline S-1 & 0 & 8.9 & 30 & 4.5 \\
S-2 & 100 & 9.2 & 34 & 6.5 \\
S-3 & 1,000 & 13.4 & 37 & 9.7 \\
S-4 & 5,000 & 18.2 & 45 & 12.2 \\
S-5 & 10,000 & 28.8 & 62 & 18.1 \\
\hline
\end{tabular}




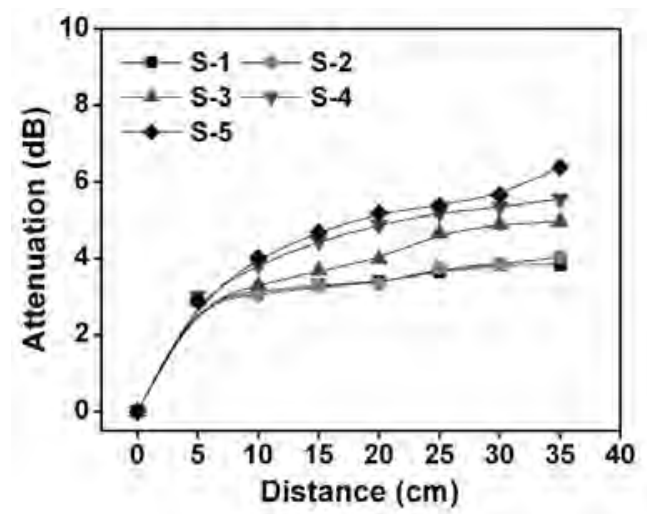

Fig. 6. Comparison of sound attenuation in differently aged transformer oils.

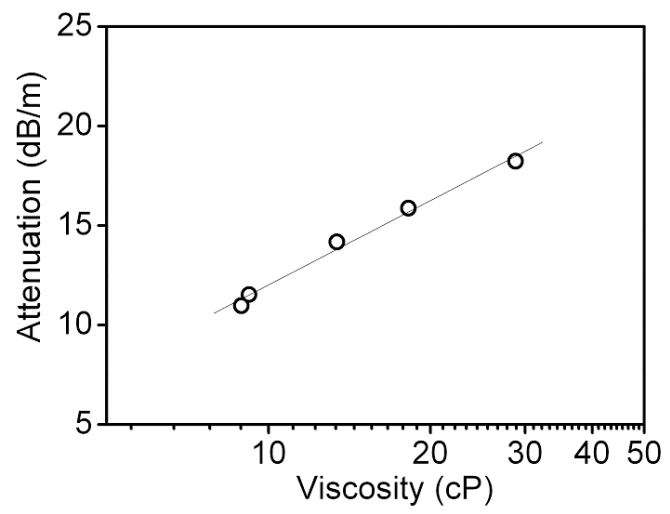

Fig. 7. Plot of sound attenuation versus viscosity of aged transformer oil.

dominated by the viscosity of the liquids. ${ }^{(14)}$ Therefore, it is speculated that the increase in oil viscosity also plays an important role in causing a higher level of acoustic wave attenuation in the aged oil.

To provide deeper insights into this relationship, the measured acoustic signal was plotted as a function of oil viscosity in Fig. 7. It was found that the acoustic wave attenuation expressed in $\mathrm{dB} / \mathrm{m}$ has a linear relationship with oil viscosity plotted on a logarithmic scale. It should be noted here that there may be some other factors that cause the correlation between the condition of the oil and the acoustic attenuation properties. However, these results show that the observation of acoustic attenuation in oil with a fiber optic sensor can indicate the amount of degradation of the transformer oil. 


\section{Conclusions}

Conventional test methods for evaluating the condition of transformer oil have certain limitations. To overcome such problems, we have proposed the use of fiber optic sensors for monitoring the condition of transformers.

We have demonstrated that the fiber optic sensor in the present work can respond suitably to the acoustic waves propagating in the transformer oil. It was observed that the sound attenuation measured using the fiber optic sensor increases as the aging process of transformer oil proceeds. Even though a detailed work is necessary to determine the correlation between the measured signals and the extent to which the transformer oil has aged, it is shown that the aging of transformer oil can be detected by fiber optic sensors.

\section{References}

1 D. W. Crofts: IEEE Trans. Electrical Insulation 23 (1988) 137.

2 S. A. Boggs: IEEE Electrical Insulation Magazine 6 (1990) 33.

3 C. Bengtsson: IEEE Trans. Power Delivery 11 (1996) 1379.

4 M. G. Danikas: IEEE Electrical Insulation Magazine 6 (1990) 27.

5 M. Wang: IEEE Insulation Magazine 18 (2002) 12.

6 A. M. Emsley: IEE Proc. Science, Measurement, and Technology, 1994, p. 324.

7 T. O. Rouse: IEEE Electrical Insulation Magazine 14 (1998) 6.

8 V. G. Arakelian: IEEE Electrical Insulation Magazine 18 (2002) 26.

9 V. Sokolov: Electrical Insulation Conference and Electrical Manufacturing \& Coil Winding Conference 1999 (Cincinnati, OH, USA, 1999) p. 659.

10 M. D. Judd: Power Eng. J. 16 (2002) 297.

11 T. Y. Kim: IEEE Trans. DEI 10 (2003) 266.

12 G. C. Stone: IEEE Electrical Insulation Magazine 7 (1991) 9.

13 L. E. Lundgaard: IEEE Electrical Insulation Magazine 8 (1992) 34.

14 L. E. Lundgaard: IEEE Electrical Insulation Magazine 8 (1992) 25. 